\title{
The frequency of polymorphic variants of filaggrin gene and clinical atopic dermatitis
}

\author{
Agata Filipowska-Grońska ${ }^{1}$, Maria Weryńska-Kalemba ${ }^{1}$, Andrzej Bożek ${ }^{1}$, Barbara Filipowska ${ }^{1}, J_{d}$ dwiga Żebracka-Gala ${ }^{2}$, \\ Dagmara Rusinek², Dorota Kula², Jerzy Jarząb ${ }^{1}$
}

${ }^{1}$ Chair and Clinical Department of Internal Medicine, Dermatology and Allergology in Zabrze, Silesian Medical University in Katowice, Poland

2Department of Nuclear Medicine and Endocrine Oncology, Institute of Oncology, Gliwice Branch, Poland

Adv Dermatol Allergol 2016; XXXIII (1): 37-41

DOI: $10.5114 /$ pdia.2015.48036

\begin{abstract}
Introduction: As far as pathogenesis of the atopic dermatitis (AD) is concerned, the roles of an impaired epidermal barrier and cornified cell envelope are widely emphasized.

Aim: The assessment of mutations of the filaggrin gene and their connection with the clinical picture of AD as well as selected allergological and environmental indicators.

Material and methods: 105 patients with diagnosed AD on the basis of diagnostic criteria were included. For every patient of the examined group, quantitative determination of the total concentration of IgE and the concentration of IgE antibodies to selected allergens were examined. For all patients, studies were performed by means of analysis of two genomic gene variants of profilaggrin (FLG) - R501X and 2282del4.

Results: Loss-of-function mutations in the filaggrin gene were shown in 12 (11.4\%) patients in the examined group. All patients in the study group who developed one of the tested loss-of-function mutations in the filaggrin gene demonstrated an extrinsic, allergic form of atopic dermatitis. A significant association $(p=0.0002)$ between the presence of one of the tested loss-of-function mutations in the filaggrin gene and elevated levels of total concentration of immunoglobulin E was shown.

Conclusions: Patients with AD of null mutations in the filaggrin gene demonstrate a relationship with the total and specific concentration of immunoglobulin E, specifically higher concentrations of IgE against aeroallergens and alimentary allergens as well as elevated levels of total immunoglobulin E.
\end{abstract}

Key words: atopic dermatitis, filaggrin, skin barrier, mutation.

\section{Introduction}

Hereditary disorders of the structure and function of the epidermal barrier as well as disorders of primary and secondary immunity system responses which interact with infectious and environmental factors are the essence of the pathophysiological basis of atopic dermatitis (AD) [1-3].

Filaggrin with keratin is the main component of the stratum corneum cells (80-90\%). Its precursor is rich in histidine, highly phosphorylated polypeptide-profilaggrin, which is the main component of the granularity of keratohyalin granular layer of epidermis [4]. Filaggrin metabolism is associated with an acidic $\mathrm{pH}$ of the stratum corneum, which is the optimal environment for the enzyme synthesizing lipids, and also contributes to the so-called natural moisturizing factor (NMF). The NMF is composed principally of free amino acids, and various derivatives of these amino acids such as pyrrolidone carboxylic acid (PCA) (which are degradation products of filaggrin) urocanic acid (a natural absorber of ultraviolet (UV) light), and inorganic salts, sugars, as well as lactic acid and urea [5]. It has been shown that the metabolites of filaggrin such as urocanic acid (UCA) and PCA reduce skin colonization by Staphylococcus aureus. In addition, PCA concentration in the stratum corneum was found to be a possible biomarker of FLG genotype [6, 7]. Gan et al. in as early as 1990 found that depending on the source of the genomic DNA, a gene contains 10,11 or 12 repetitions of the filaggrin unit, followed by a gene family segregation in accordance with the established

Address for correspondence: Agata Filipowska-Grońska MD, PhD, Chair and Clinical Department of Internal Medicine, Dermatology and Allergology in Zabrze, Silesian Medical University, 13-15 3 Maja St, 41-800 Zabrze, Poland, phone: +48 603121817 ,

e-mail: agata.filipowska@wp.pl

Received: 19.01.2014, accepted: 10.07.2014. 
laws of Mendelian inheritance. This means that the human profilaggrin arrangement of genes is polymorphic due to genetic variability between individuals [8,9]. At present more than 40 types of mutations of allelic variants of the filaggrin gene have been described. A connection between filaggrin gene mutation with the early occurrence of the disease and its persistence into adulthood was discovered [10]. It is also believed that although not in all patients (approximately 25-50\%), the above-described FLG mutations occur, in the remaining patients at the root of AD there may be other congenital or acquired defects in filaggrin [11]. The consequences of the filaggrin deficiency was analyzed in many works, not only with regard to $A D$, but also ichthyosis vulgaris and other atopic diseases [12].

\section{Aim}

The aim of this study was to assess the frequency of the loss-of-function mutations in the filaggrin gene and its relationship in the clinical picture of $A D$.

\section{Material and methods}

The studied group consisted of 105 adults of both sexes (65 women, 40 men) with a mean age of $37 \pm 17.38$ with $A D$.

The subjective and objective examinations of patients and immunological studies were performed in the Clinical Department of Internal Medicine, Dermatology and Allergology in Zabrze. Genetic analysis of FLG gene polymorphisms was carried out at the Laboratory of Molecular Diagnostics and Functional Genomics Department of Nuclear Medicine and Endocrine Oncology, Institute of Oncology, Gliwice Branch. The diagnosis of AD was based on generally accepted Hanifin and Rajka criteria. All patients were asked about medical personal and familial history, with respect to atopic diseases. Furthermore, each patient under the supervision of the investigator filled in the questionnaire, taking into account factors that modulate the course of the disease. The extent and severity of skin lesions was rated by SCORAD index (SCORingAtopicDermatitis) [13]. The total concentration of IgE in serum using the ELISA immunoassay method with an Allergopharm kit was determined in each of the patients from the examined group. The analysis of FLG R501X gene polymorphism was based on the reaction of allelic discrimination in 7900HT Fast Real-Time PCR System from Applied Biosystems. 2282del4 deletion analysis was performed by genotyping using a fragment length analysis sequencer 3130 Genetic Analyzer from Applied Biosystems using Genescan software.

History positive for atopy was noted in 77 (73\%) patients. Food allergy in childhood occurred in 27 (26\%) patients, atopic asthma in 31 (30\%) subjects, allergic rhinitis was present in 47 (45\%) subjects, urticaria symptoms in
20 (19\%) patients, while allergic conjunctivitis in 37 (35\%) subjects. Based on the survey, the severity of pruritus and factors aggravating it were analyzed. Thirty-four (32\%) individuals identified the severity of pruritus as minor - grade 1, 32 (30\%) people as moderate - grade 2 , while in 39 (37\%) patients intense severity of pruritus occurred - grade 3. In 60 (57\%) subjects sleep disturbances associated with itching were noted. Forty-eight (45.7\%) people in the study group reported the exacerbation of pruritus in contact with wool.

The course of the disease was evaluated in the examined group of patients. Periods of remission longer than three months during the year (1 point) were observed in $37 \%$ of patients (39 patients), periods of remission of less than three months within a year (2 points) were also observed in $37 \%$ of patients (39 patients), solid persistence of changes ( 3 points) occurred in $26 \%$ of patients ( 28 patients). Thirty-seven percentage of the respondents had dry skin symptoms, $25 \%$ showed lichenification of the skin, in $23 \%$ of patients, among skin disorders erythema was demonstrated. Symptoms of more active disorders such as excoriations, edema/papules, oozing/crusting occurred in 5\% of the respondents, $19 \%$ and 3\%, respectively. Analyzing the distribution of skin lesions, the following values were obtained: in 91\% of subjects lesions around the elbow and knees areas were found, disseminated lesions, located on the limbs, trunk, but with a mediocre severity occurred in $56 \%$ of patients, changes on the hands were observed in $49 \%$ of patients, in $33 \%$ of patients it appeared on the face, scalp head was affected in $12 \%$ of the patients, erythroderma occurred in $6 \%$ of patients. The factors which modulate the course of AD were analyzed. Skin lesions were worsened by stressful situations in $57 \%$ of patients, an increased incidence of various types of viral, bacterial and parasitic infections were found in $34 \%$ of the patients, co-existing conditions were present in $28 \%$ of patients, and allergy to nickel was reported in an interview by $38 \%$ of patients.

\section{Results}

Analysis of the loss-of-function mutations in the filaggrin gene 2282del4 mutation, involving the deletion of 4 pairs of alkalis at position 2282 on the third exon of the filaggrin gene in both alleles (homozygote AA) was present in 3 patients in the study group (2.86\%); there were 7 (6.67\%) patients with mutations in one allele (heterozygote $A B$ ). The remaining patients (95 people - 90\%) showed no mutations in both alleles (homozygote BB). The total number of persons with at least one mutant allele $2282 \mathrm{del} 4$ in the filaggrin gene that conditions the loss-of-function of the product was 10 (9.5\%). The total number of persons with at least one mutant allele in the range of R501X polymorphism in the filaggrin gene that conditions the loss of the function of the product was 2 (1.9\%). The total number of the examined group 
with at least one type of loss-of-function mutation was $12(11.4 \%)$ - Figure 1.

The median of total IgE was $312 \mathrm{kU} / \mathrm{l}$ (range: 11, 13678) in the study group. The median of total IgE in patients with the loss-of-function mutation was significantly higher at $2500 \mathrm{kU} / \mathrm{l}$. The range of values was between 316 and $13678 \mathrm{kU} / \mathrm{l}$. The median of total IgE in patients without any loss-of-function mutations was lower than the value in the whole group and was $224 \mathrm{kU} / \mathrm{l}$, with a range between 11 and $13311 \mathrm{kU} / \mathrm{l}$. The difference between the groups was highly statistically significant in MannWhitney $U$ test $(p<0.0002)$ (Figure 2 ).

In the study group of people with one of the two lossof-function mutations (11.4\%), lesions covered more than $30 \%$ of the body surface with $8 \%$ of patients representing $70 \%$ of all subjects with the "null" mutation.

The median of SCORAD index was 45 (range: 10, 87) in the study group. This value of the index corresponds to the degree of severity of symptoms in a moderate form of AD. The median of SCORAD index in patients with a loss-of-function mutation was significantly higher at 69 , with a range between 21 and 87 , as to define the degree of severity of symptoms of a severe form of AD. The median of SCORAD index in patients without any loss-of-function mutation was similar to the value in the whole group - 43 (range: 10, 87). The difference between the groups was highly statistically significant in MannWhitney $U$ test $(p<0.001)$ (Figure 3$)$.

\section{Discussion}

Still growing incidence of AD observed in recent years has led to more and more accurate, multi-population research being carried out. It has brought a lot of interesting information on the mechanisms of epidermal barrier defects. A breakthrough in the research of the filaggrin gene mutations was made with the discovery of the importance of filaggrin gene (FLG) in the pathogenesis of atopic eczema by Palmer et al. Their work, which was published in 2006, clearly showed a link between mutations R501X as well as 2282del4 and AD [9]. It was estimated that a loss-of-function mutation in the filaggrin gene was $9 \%$ in the European population.

In the present study, loss-of-function mutations in the filaggrin gene were found in $11.4 \%$ of patients of the tested group, however, it should be emphasized that this value is close to the frequency (9.96\%) shown by Narbutt et al. in the Polish population [14]. Due to the small size of the study group, this value should only be treated as probably characterizing the Polish population. Improving genotyping methods and a strict selection of patients in the study group, allow for a more accurate determination of the percentage of mutant alleles in the studied populations, which may explain a lot lower score (4.71\%) obtained by Kowalewski et al. in 2007 [15]. This is also the case among the Irish, Danish and Scottish authors

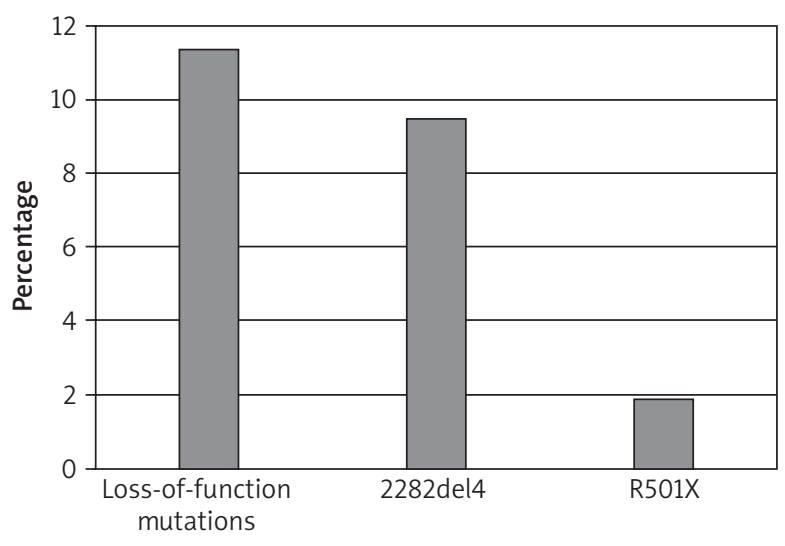

Figure 1. Loss-of-function mutations in the examined group

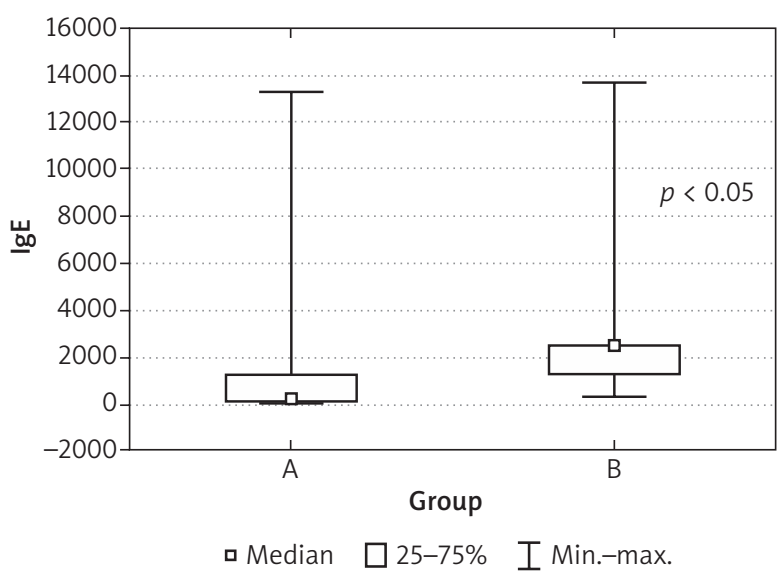

Figure 2. The concentration of the total IgE depending on the presence of a loss-of-function mutation: group $A$ - without loss-of-function mutations, group $B$ - with lossof-function mutations

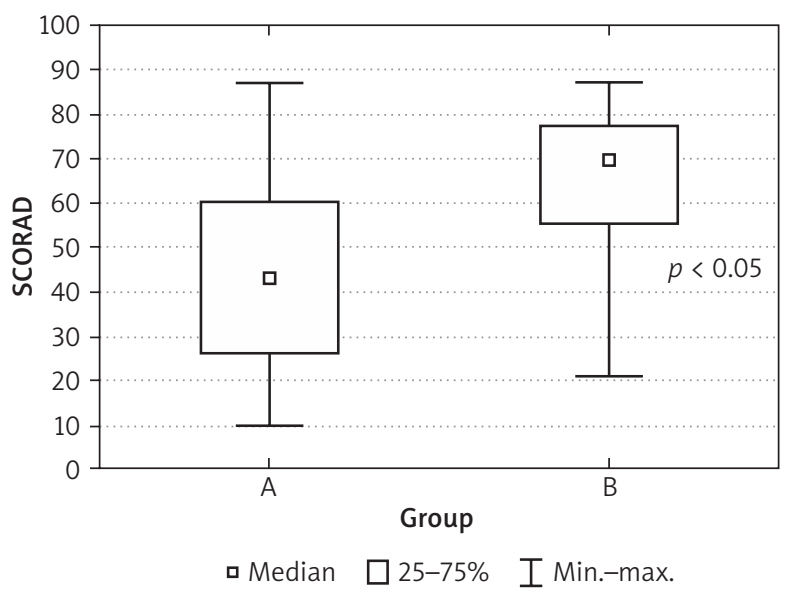

Figure 3. The SCORAD index: group A - without loss-of-function mutations, group $B$ - with loss-of-function mutations 
where obtained prevalence of the studied mutations is lower [16]. However Henderson et al. study on a large group of the British children (ALPS) has confirmed that approximately $15 \%$ of cases of AD with severity of symptoms are associated with the occurrence of at least one of said mutations [17].

Comparing the two studied polymorphisms, R501X substitution variant has been shown only in $1.9 \%$ of patients in the study group, while 2282del4 deletion was found in $9.5 \%$ of patients. The resulting difference is comparable with other studies evaluating the Polish population $[14,15]$ and indicates the presence of a lower percentage of R501X polymorphism.

In the paper, a correlation $(p<0.05)$ between the occurrence of one of the tested loss-of-function mutations in the filaggrin gene and elevated levels of total immunoglobulin E were found. Furthermore, in all patients with a polymorphism of the tested gene, an extrinsic, allergic form of atopic eczema has been shown. These types of observations are identical to the results of large population-based studies ISAAC II and ALPSAC [17, 18]. The ISAAC II study demonstrated the presence of R501X mutation and 2281del 4 together with the increased predisposition to specific sensitization as assessed by positive prick tests, and an increase in specific IgE antibodies.

The study achieved significant relation $(p<0.05)$ between high SCORAD index scores and the occurrence of loss-of-function mutation. Among the people with the demonstrated mutation in the filaggrin gene, the score of SCORAD was observed at moderate and considerable degrees.

It seems necessary to continue the studies and analysis of the relationship between these polymorphisms and the course of the disease, with regard to various clinical stages of the disease. Selected parameters of the epidermal barrier function of the skin as transepidermal water loss (TEWL) or severity of erythema characterize the severity of AD. The measurement of the loss of the water from the epidermis in correlation with the scale of severity of itching could be helpful in demonstrating the phenotypic nature of the filaggrin gene defect [19]. It seems important to extend the study by adding the data on the course of the disorder in the first years of the patient's disease with mutations in the filaggrin gene defect life [20].

The epidermal barrier plays a key role in immune surveillance and homeostasis, in preventing the penetration of the products of microorganisms and allergens [21].

A multicenter comparison study could be helpful in demonstrating the phenotypic nature of the filaggrin gene defect, and an additional analysis of the interaction of the environmental factors and other genetic factors would provide more accurate knowledge of such interesting and complex disease, which undoubtedly $A D$ is.

\section{Conclusions}

The frequency of the loss-of-function mutation in the filaggrin gene is comparable or slightly more frequent than in other studies. In all patients with a polymorphism of the tested gene, an extrinsic, allergic form of atopic eczema has been shown. This is similar with that observed in other large population analyses.

\section{Conflict of interest}

The authors declare no conflict of interest.

\section{References}

1. Placek W. Genetyczne uwarunkowania atopowego zapalenia skóry. Postep Derm Alerg 2010; 27: 325.

2. Nedoszytko B, Sokołowska-Wojdyło M, RuckemannDziurdzińska K, et al. Chemokines and cytokines network in the pathogenesis of the inflammatory skin diseases: atopic dermatitis, psoriasis and skin mastocytosis. Adv Dermatol Allergol 2014; 31: 84-91.

3. Rutkowski K, Sowa P, Rutkowska-Talipska J, et al. Allergic diseases: the price of civilisational progress. Adv Dermatol Allergol 2014; 31: 77-83.

4. McGrath JA. Filaggrin and the great epidermal barrier grief. Australas I Dermatol 2008; 49: 67-73.

5. Brown SJ, Irvine AD. Atopic eczema and the filaggrin story. Semin Cutan Med Surg 2008; 27: 128-37.

6. Fluhr JW, Elias PM, Man MQ, et al. Is the filaggrin-histidineurocanic acid pathway essential for stratum corneum acidification? J Invest Dermatol 2010; 130: 2141-4.

7. Kezic S, Kammeyer A, Calkoen F, et al. Natural moisturizing factor components in the stratum corneum as biomarkers of filaggrin genotype: evaluation of minimally invasive methods. Br J Dermatol 2009; 161: 1098-104.

8. Gan SQ, McBride OW, Idler WW, et al. Organization, structure, and polymorphisms of the human profilaggrin gene. Biochemistry 1990; 29: 9432-40.

9. Palmer CNA, Irvine AD, Terron-Kwiatkowski A, et al. Common loss-of-function variants of the epidermal barrier protein filaggrin are a major predisposing factor for atopic dermatitis. Nat Genet 2006; 38: 441-6.

10. Irvine AD. Fleshing out filaggrin phenotypes. J Invest Dermatol 2007; 127: 504-7.

11. McGrath JA, Uitto J. The filaggrin story: novel insights into skin-barrier function and disease. Trends Mol Med 2008; 14: 20-7.

12. Armengot-Carbo M, Hernández-Martín A, Torrelo A. The role of filaggrin in the skin barrier and disease development. Actas Dermosifiliogr 2015; 106: 86-95.

13. Severity scoring of atopic dermatitis: the SCORAD index. Consensus Report of the European Task Force on Atopic Dermatitis". Dermatology (Basel) 1993; 186: 23-31.

14. Narbutt J, Geel M, Sysa-Jędrzejowska A, Stelmach I. R501X and 2282del4 mutations in filaggrin gene in Polish population of AD patiens. Dermatology and Venereology. 6-10 October 2010, Gotheborg, Sweden.

15. Kowalewski C. Structure and function of the epidermal barrier and its disorders in atopic dermatitis. Allergology.org. September 2007.

16. Sandilands A, Terron-Kwiatkowski A, Hull PR. Comprehensive analysis of the gene encoding filaggrin uncovers preva- 
lent and rare mutations in ichthyosis vulgaris and atopic eczema. Nat Genet 2007; 39: 650-4.

17. Henderson J, Northstone K, Lee $\mathrm{S}$, et al. The burden of disease associated with filaggrin mutations: a populationbased, longitudinal birth cohort study. J Allergy Clin Immunol 2008; 121: 872-7.

18. Weidinger S, O'Sullivan M, Illig T, et al. Filaggrin mutations, atopic eczema, hay fever, and asthma in children. J Allergy Clin Immunol 2008; 121: 1203-9.

19. Polańska A, Dańczak-Pazdrowska A, Silny W, et al. Evaluation of selected skin barrier functions in atopic dermatitis in relation to the disease severity and pruritus. Adv Dermatol Allergol 2012; 29: 373-7.

20. Ogrodowczyk A, Wróblewska B, Markiewicz LH, et al. Possible immunological consequences of filaggrin gene mutation. A case study of a 3-year-old allergic girl. Adv Dermatol Allergol 2013; 38: 403-7.

21. Agrawal R, Woodfolk JA. Skin barrier defects in atopic dermatitis. Curr Allergy Asthma Rep 2014; 14: 433. 